\title{
The Symmetric Paradigm
}

\section{I.I The Symmetric Paradigm}

From grade school to the higher reaches of tense logic, a fundamental paradigm prevails. Human languages offer up the ability to talk about past events, present ones, and future ones. According to the paradigm, in many human languages, this is implemented by a system of tenses with three broad categories: past, present, and future. With some exceptions and complications, these tenses line up with the temporal location of the appropriate events. ${ }^{\text {I }}$

The insight that past and future tenses have symmetric meanings is central to research frameworks that build on the classical framework for the logic of tense (Prior, 1957, 1967, 1969). The symmetric paradigm reverberates in many theoretical decisions concerning the syntax, the semantics, and logic of discourse about the future. When Prior (1967, p. 35) provides a series of postulates for "the logic of futurity," he immediately proceeds to lay down "a series of analogous postulates ... to give the logic of pastness." Fast forward fifty years, and we find that von Fintel and Heim (2OII) take as a starting point an approach on which the semantic entries for future and past tense (lifted from tense logic) look like "mirror images of each other, though they are of different syntactic categories" (p. 72).

This chapter develops the core idea of the symmetric paradigm. Eventually, I will critique this idea, with an eye toward developing my own, nonsymmetric account of future discourse. But before any cards are on the table, I want to clarify that my critique of the symmetric paradigm is limited in scope. Even if it turned out that the meanings of the expressions we use to talk about the future - the predictive expressions, as I will say are of an entirely different sort from the meanings of the expressions we recruit to talk about the past, there is nothing inherently objectionable

I While some languages, such as Mandarin Chinese, lack tense markers, it is nevertheless possible to characterize their devices of temporal reference symmetrically (Bittner, 20I4). 
about designing a system of logic with symmetric tenses. This is because studying the logic of tensed statements need not be part of a model of the meaning of tense in natural language. A formal temporal logic could be part of an attempt to regiment certain philosophical arguments and to clearly display their structural features. Alternatively, its purpose could be to state and compare rules for automated temporal reasoning - the kind of rules that we might want to instill in a computer carrying out reasoning tasks involving temporally structured information. ${ }^{2}$

A formal framework only becomes exposed to empirical evidence, and to the sort of argumentation I will build against the symmetric paradigm, if it is embedded in a theory with the ambition of predicting linguistic phenomena. This is the case for natural language semantics. These explanatory ambitions include developing a model of temporal discourse that systematizes and explains our judgments of acceptability (or unacceptability) of various speech acts in context as well as our judgments about the acceptability (or unacceptability) of various inference patterns.

With that said, even if Prior himself might not have had natural language applications on his mind, the interpretation of the tense logic framework as a module in a theory of meaning has nonetheless been influential. For example, simple tense logic is the default model of tense that is developed in two of the most influential textbooks in natural language semantics (Dowty et al., 198I, and in the notes in von Fintel and Heim, 20II) as well as in Richard Montague's influential essay "Pragmatics" (which can now be found in Montague, 1974, pp. 95-II9). Even those who have objected to this application of tense logic have often landed on symmetric analyses (we will see some examples in due course).

Our first task, then, is to develop with more precision the hypothesis that the tenses of a natural language such as English are semantically symmetric. To do so, I spell out in detail the behavior of the simplest tense operators of tense logic. Once the symmetry assumption is spotted in this context, it is easy to also identify it in a variety of alternative, more complex frameworks.

\section{I.2 Symmetric Semantics}

Consider a toy language capable of expressing temporally structured information. The core feature of the sort of language I have in mind is that basic tensed sentences are the product of composing a temporal operator

2 See, e.g., Goldblatt (2006), $\$ 7.3$ on the logic of concurrency. 
with a tenseless sentence-like object. Thus the sentence she passed might be the result of composing a tense operator (something with the meaning of in the past) and a tenseless core (she pass). (Note that in giving examples of such tenseless cores, I will omit gender and number features.) It is a substantive empirical assumption, though one that seems initially plausible for many languages, that natural language sentences have this kind of linguistic structure. At any rate, I will accept this assumption for the sake of illustration.

The basic building blocks of the toy language are sentence radicals. Radicals are tenseless descriptions of events (Bea run) or states (Al be happy). I will not explore the inner structure of tense radicals, assuming instead that they are directly interpretable for truth and that if they are not in the scope of a tense operator, they default to a present interpretation. So Al be happy describes a state of happiness by $\mathrm{Al}$ that happens at the time at which it is uttered. (As the semantic development approaches something closer to English, in Chapter 7, I will require that radicals always combine with tense before being even interpretable for truth or falsity.) In addition to radicals, our toy language contains Boolean connectives (and, or, not, etc.) and the temporal operators was and will.

In this language, one can say things with obvious English analogues such as

\section{will(Bea run) and not was (al be happy)}

The temporal operators of the toy language have meanings that roughly translate as at some point in the past and at some point in the future.

We can already read off these informal glosses the expectation that the meanings of was and will should be symmetric. This expectation is borne out upon development of standard techniques of model-theoretic semantics. ${ }^{3}$

The first thing we need toward that development are, of course, models. Think of models as abstract objects that depict the features of reality that are needed to capture the semantic properties of the expressions of the language. To use Etchemendy's (1990, chapter 2) terminology, this means that models are understood representationally. That is to say, models are simplified representations of temporally structured worlds. Under this conception, two different models represent two different ways a temporally

3 A word about notation: When ignoring what happens at a sub-sentential level, I generalize over sentences and sentence radicals of this language by means of variables such as A, B, C, etc. In these cases, I will only be concerned with connectives and temporal operators as means of composition of sentence radicals. 
structured world could be - fixing the meanings of our words. ${ }^{4}$ Needless to say, much as our toy language does not aim to represent all the complexities of a natural language, these models incorporate substantial idealizations. They do not aim to represent all the complexity of our world and they do not aim to be completely accurate representations of those features that they do represent. With these clarifications in mind, define:

Definition I.I A model $\mathcal{M}$ is a triple $\langle\mathcal{T},<, v\rangle$ with $\mathcal{T}$ a set of times; $<$ a linear order on $\mathcal{T}$; and $v$ a valuation function.

A linear order on a set $S$ is a transitive, antisymmetric, and total relation on $S$. The valuation function $v$ maps each sentence radical of the target language $\mathcal{L}$ to a truth-value relative to a time. So if Bea does run at $t_{0}$, we have $v\left(\right.$ Bea run,$\left.t_{0}\right)=\mathrm{I}$. The possible truth-values in our interpretation schema are $\mathrm{I}$, for true, and o, for false.

Suppose that our toy language contains exactly four radicals

Bea run, Al run, Bea be happy, Al be happy

Figure I.I diagrams the structure of a simple model and possible assignments to sentence radicals by the valuation function. In these simplified settings, we can diagram the valuation function at each point by a sequence of four o's and I's, where a I in the $n$th place of the sequence means that the $n$th radical (in the preceding list) is true at the given time, and a o means that it is false. In the model diagrammed by Figure I.I, Bea and $A l$ are both running and happy at $t_{2}$; they both stop running at $t_{3}$, but only $A l$ is happy then, and he stays happy for one more tick of the clock. After that, the world ends.

Models have two jobs: to contribute to a theory of the truth-conditions of sentences of the language and to contribute to an account of logical entailment. However, models (as just characterized) are not enough to state the truth-conditions for the sentences that are of interest. The question whether Bea be happy is true in the model of Figure I.I is ill posed: Bea is happy at some but not all times. To determine whether the radical Bea



Figure I.I Valuation function for a model with four radicals

4 Representational models contrast with "interpretational models," which represent different ways in which we might interpret the nonlogical fragment of the language fixing what the world is like. 
be happy is true, one must take the perspective of a particular point in the temporal development of the model. ${ }^{5}$ Speaking formally, the compositional semantics for this language centers not on a definition of truth in a model $\mathcal{M}$ but on a definition of truth at a point of evaluation. In the semantics for the toy language, a point of evaluation consists of a model together with a time that is drawn from the stock of times of that model.

Given a model $\mathcal{M}$ and a time $t$ that belongs to the model's stock of times, truth-values (again, I for true and o for false) can be assigned to all sentences of the language. The double brackets $\llbracket \cdot]^{\prime}$ ' denote the interpretation function. This function inputs an expression and a point of evaluation and outputs the semantic value at a point of evaluation. Since points of evaluation are pairs consisting of a model and a time, I will write $\llbracket \cdot \rrbracket^{\mathcal{M}, t}$. As our points of evaluation change, so will the parameters in the double-bracket notation. Unless it is important to remind the reader that everything is relativized to a model, I omit the $\mathcal{M}$ superscript.

The assignment starts with sentence radicals (this language's equivalent of "atomic sentences") that are interpreted directly by the valuation function. Suppose we want to evaluate the semantic value of Bea run at $t_{\mathrm{I}}$ in model $\mathcal{M}$. First, extract the model's valuation function $v_{\mathcal{M}}$, and then check what the valuation function assigns to Bea run at $t_{\mathrm{I}}$. In the model of Figure I.I, this is o, so we write $\llbracket$ Bea run $\rrbracket^{t_{1}}=0$.

The pattern of propagation is determined by the lexical entries of the various expressions that are used to generate complex sentences. For sentential connectives, adopt standard Boolean entries.

$$
\begin{aligned}
& \llbracket \mathrm{A} \text { and } \mathrm{B} \rrbracket^{t}=\min \left(\llbracket \mathrm{A} \rrbracket^{t}, \llbracket \mathrm{B} \rrbracket^{t}\right) \\
& \llbracket \mathrm{A} \text { or } \mathrm{B} \rrbracket^{t}=\max \left(\llbracket \mathrm{A} \rrbracket^{t}, \llbracket \mathrm{B} \rrbracket^{t}\right) \\
& \llbracket \operatorname{not} \mathrm{A} \rrbracket^{t}=\mathrm{I}-\llbracket \mathrm{A} \rrbracket^{t}
\end{aligned}
$$

A conjunction is true just in case both its conjuncts are true; a disjunction is true if even one disjunct is; and negations flip truth-values around.

Now for the queen of the entries. The goal is to assign semantic values to was and will that capture the symmetric approach to truth-conditions of past and future claims. To this end, say that was(Bea run) is true at $t$ if there is a prior time at which Bea runs; on the future side, say will(Bea run) is true at $t$ if there is a future time at which Bea runs. Figure I.2 illustrates the idea.

5 This parallels one of the standard moves to interpret models for modal logic: In that context, the points at which we evaluate atomic sentences are possible worlds. If the worlds in a model disagree on the truth-value of some sentence A, and for whatever reason it matters to fix that truth-value, we can designate a world in the model as actual. 


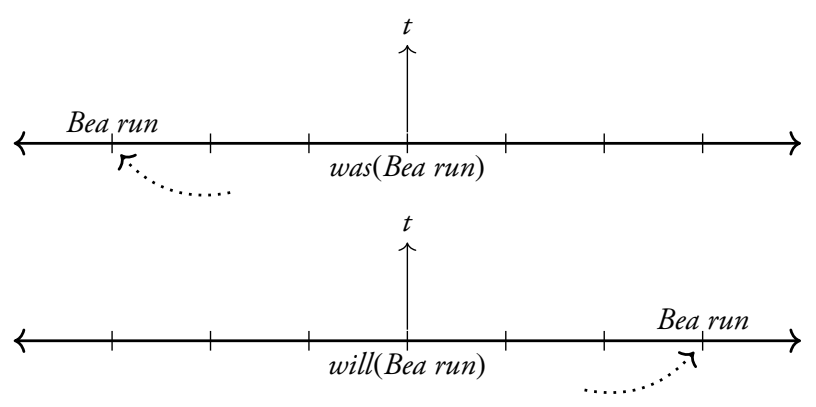

Figure I.2 Temporal shift for symmetric semantics

More formally, and more generally, we can state the following pair of lexical entries:

LINEAR SYMMETRIC SEMANTICS

a. $\llbracket$ was $(\mathrm{A}) \rrbracket^{t}=\mathrm{I}$ iff $\exists u<t, \llbracket \mathrm{A} \rrbracket^{u}=\mathrm{I}$

b. $\quad \llbracket w i l l(\mathrm{~A}) \rrbracket^{t}=\mathrm{I}$ iff $\exists u>t, \llbracket \mathrm{A} \rrbracket^{u}=\mathrm{I}$

These entries complete the definition of truth at a model/time pair for our toy language.

From the model-theoretic perspective, a near-classical way to define entailment is as preservation of truth at a point of evaluation (a pair $\langle\mathcal{M}, t\rangle)$. An argument with premises $A_{I}, \ldots, A_{n}$ and conclusion $C$ is valid (written $A_{1}, \ldots, A_{n}=C$ ) just in case there is no model $\mathcal{M}$ and time $t \in \mathcal{T}_{\mathcal{M}}$ such that all the $\mathrm{A}^{\prime}$ s are true at $\langle\mathcal{M}, t\rangle$ but $\mathrm{C}$ is not. It is invalid otherwise (in which case, we write $A_{I}, \ldots, A_{n} \not \models C$ ).

The system of logic resulting from this interpretation of temporal language has been studied in A. N. Prior's seminal work (Prior, 1957, 1967, 1969) and, later, in the context of the explosion of model theoretic techniques for modal logic (Rescher and Urquhart, 197I; Burgess, 1979). The appendix to this chapter reviews a sound and complete axiomatization of (a slight refinement on) this consequence relation.

At a less formal level, it is important to understand the proper interpretation of verdicts about validity and invalidity in this system. Here are four sample patterns, a valid one and three invalid ones.
a. $\quad$ will $($ will $(\mathrm{A}))=$ will $(\mathrm{A})$
b. $\quad \mathbf{A} \not \models$ will $(\mathrm{A})$
c. $\quad \mathbf{A} \not \models$ will $($ was $(\mathbf{A}))$
d. $\quad \mathbf{A} \not \models$ was $($ will $(\mathbf{A}))$ 
It is tempting to assess these verdicts by considering parallel inferences in English and evaluating whether they sound valid. It is remarkable, however, that the inferences in (I) do not have immediate, natural-sounding translations. Consider (I-d). The tempting thought is to view this as a theoretical prediction about the invalidity of an inference that in English we might put as follows:

\section{I am happy}

\section{It was the case at some point in the past that I was going to be happy}

The problem is that the English inference features additional material that is not exactly semantically inert: was going to is not precisely the same as will. Another problem is that, as I will show shortly, even holding fixed the semantics, we can change the validities by imposing constraints on the class of models. For this reason, we need to be careful in making judgments about the acceptability of this inference as evidence for or against the semantics.

It is possible to justify the verdicts in (I) by appealing to intuitions of a different sort. We might have intuitions about whether these inferences should come out valid given the informal glosses for the tense operators. To illustrate with $(\mathrm{I}-\mathrm{a})$, we might reason that if at some future point, there is a further future point at which $A$ is true, then it has to be true that at the origin point there is a future point at which $A$ is true. As for $(\mathrm{I}-\mathrm{b})$, it is clearly invalid under the informal glosses we have been operating with: The present truth of $A$ at some point in time is not enough to establish the truth of $A$ at a later point. While these judgments cannot be used to address the empirical adequacy of the symmetric semantics, they can help us investigate whether the formalism is a correct implementation of its informal design specifications.

In addition, the semantics can illuminate correspondences between logical validities and the temporal reality they represent. Consider again (I-c) and (I-d). As we think through the meanings of the symmetric tense operators, we might intuit (I-C) and (I-d) as valid. Surprisingly, the current semantics invalidates them both. Consider evaluating Al be happy at the last time $t^{*}$ of a finite model. Because this is the last time in the model, every sentence of the form will $\mathrm{A}$ is false at $t^{*}$. After all, if there is no time after $t^{*}$, there is no time after $t^{*}$ at which $\mathrm{A}$ is true. As a result, both will(Al be happy) and will not(Al be happy) are false. To enforce the validity of (I-c) and (I-d), we could constrain time to have no endpoints toward the future, which validates $(\mathrm{I}-\mathrm{C})$, or toward the past, which validates $(\mathrm{I}-\mathrm{d})$. The familiar point 
here is that, given the standard tense logic entries, there is a correspondence between the metaphysical assumption that timelines are infinite and the validity of (I-C) and (I-d).

Alternatively, we could adopt a more pragmatic perspective: In any context in which speakers can convince themselves that they are not evaluating from the temporal edge of the world, these schemas will preserve truth. So in practice, we might be warranted in treating them as valid inferences even if they are not valid in full generality. This perspective might be bolstered by noting that the following are valid entailments in this system.

$$
\begin{array}{ll}
\text { a. } & \mathrm{A}, \operatorname{will}(\mathrm{T}) \models \\
\text { b. } & \mathrm{A}, \operatorname{walll}(\mathrm{was}(\mathrm{T}) \models \operatorname{was}(\operatorname{will}(\mathrm{A}))
\end{array}
$$

(Here $T$ stands in for an arbitrary tautology.) We might take this to mean that the inferences $(\mathrm{I}-\mathrm{C})$ and $(\mathrm{I}-\mathrm{d})$ should be acceptable (again, given an informal gloss on the temporal operators) whenever it is presupposed that there is a future (by implicitly accepting will $(T)$ ) or that there is a past (by implicitly accepting was $(\top)$ ).

\section{I.3 The Symmetric Paradigm Contextualized}

The semantic analysis I provided in Section I.2 exemplifies but does not exhaust the symmetric paradigm. Much pioneering work in tense logic was not explicitly addressed to the semantic analysis of natural language. And it is misleading to take Prior's work as directly providing a semantic analysis of English tenses (Ogihara, 2007, pp. 393-397). ${ }^{6}$ Moreover, the empirical hypothesis that natural language tenses might work like tense logic operators quickly found a rival approach - namely, the view that instead they work as object-language referential devices (Partee, 1973; Dowty, 1982; see King, 2003, chapter 6 for an extensive overview).

The essence of the symmetric paradigm is not a specific semantic assumption, however. Instead, it is the general idea that the semantics of

6 Ogihara discusses the tricky case of theorists such as Montague. Unlike Prior, Montague is clearly concerned with providing a model of the semantic functioning of the natural language tense system. However, Montague's methodology involves translating from English to an intermediate language like the toy language of Section I.2. It seems possible to argue that it is not a problem if the intermediate translation language is too expressive. A more significant problem is that, even when natural language tenses do stack, the truth-conditions are generally not what is predicted by the tense operator account. 
future and past tenses ought to be mirror images of each other. Even as the semantics of tense has become more nuanced and expressive, the symmetric paradigm has maintained some of its shine and theoretical grip. In this section, I consider some simple ways of refining our understanding of the semantics of tense while sticking to the central tenets of the symmetric paradigm.

One way in which the operators of tense logic are at best coarse approximations of natural language tenses is that not every future moment can matter to our evaluation of future claims and not every past moment can matter to our evaluation of past claims. Ordinarily, as speakers, we restrict attention to certain specific points in time. Sometimes we do that by explicitly restricting the relevant temporal range. If I say I cooked dinner, I arguably do not speak truly if I cooked dinner once in 1995 . In practice, I must be talking about some restricted interval of time that is easily identifiable by my conversational partners. This suggests that temporal talk has a context-sensitive dimension that is absent from my initial formulation of the symmetric clauses. What is more, these salient intervals are not controlled only by the linguistic context. They can be explicitly restricted or modified.

(3) a. Last May, Isabella visited Canada

b. In 1984, Los Angeles hosted the Olympics

We cannot correctly analyze these phrases if English past tense just meant at some point in the past and if it did not compositionally interact with these restricting expressions.

There is a natural way to enrich the symmetric semantics to provide it with this kind of flexibility. Suppose that instead of a time, the points of evaluation also keep track of a time interval. ${ }^{7}$

Definition I.2 An interval $\mathcal{I}$ is a set of times satisfying the property that for any two times $t$ and $v$ both in $\mathcal{I}$, if $t \leq u \leq v$, then $u \in \mathcal{I}$.

More succinctly, intervals are convex sets of times (with respect to the temporal precedence ordering). ${ }^{8}$

This modification allows a distinction (as in Figure I.3) between those cases in which A occurs within the designated range $I$ and those in which

7 The idea of using intervals in the semantics comes from Bennett and Partee (1972), and has been widely applied in many subsequent frameworks (Dowty, 1982; Condoravdi, 2002).

8 Proponents of intervals in temporal semantics commonly stipulate that the set of times has the cardinality of the continuum (this is the approach of Bennett and Partee, I972, p. 69). Definition I.2 is neutral on matters of cardinality. 


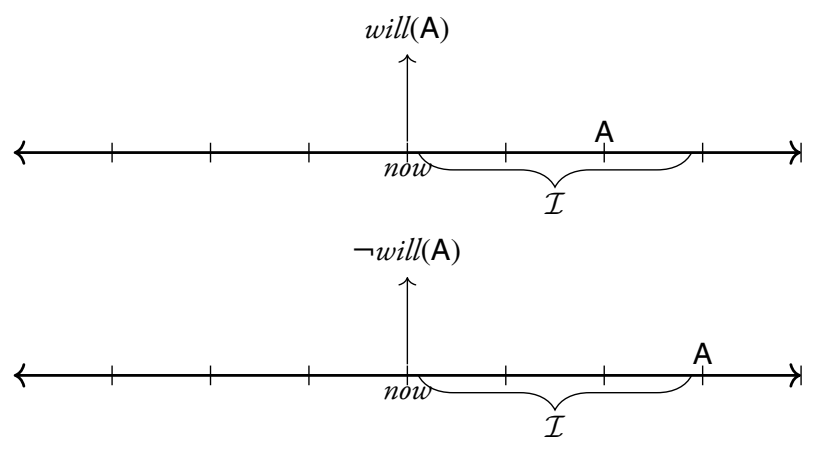

Figure I.3 Linear symmetric semantics with intervals

A occurs only outside this range. Formulating a semantics along these lines requires a different approach to the semantics for sentence radicals. But the point I want to make here is that even moving to this sort of system allows for a symmetric analysis of tense operators. Say that an interval $\mathcal{I}_{\mathrm{I}}$ precedes a $\mathcal{I}_{2}\left(\mathcal{I}_{1}<\mathcal{I}_{2}\right)$ iff every point in $\mathcal{I}_{\text {I }}$ precedes every point in $\mathcal{I}_{2}$.

LINEAR SYMMETRIC SEMANTICS WITH INTERVALS

$\begin{array}{ll}\text { a. } & \llbracket \text { was }(\mathrm{A}) \rrbracket^{\mathcal{I}}=\mathrm{I} \text { iff } \exists \mathcal{I}^{*}<\mathcal{I}, \llbracket \mathrm{A} \rrbracket^{\mathcal{I}^{*}}=\mathrm{I} \\ \text { b. } & \llbracket \text { will }(\mathrm{A}) \rrbracket^{\mathcal{I}}=\mathrm{I} \text { iff } \exists \mathcal{I}^{*}>\mathcal{I}, \llbracket \mathrm{A} \rrbracket^{\mathcal{I}^{*}}=\mathrm{I}\end{array}$

Informally, it will rain is true at an interval iff it rains is true at a later point in that interval. As noted, one would have to explain what it means for a radical to be true relative to an interval. The idea we will implement (in Chapter 7) for radicals such as it rains is that this is true relative to $\mathcal{I}$ iff there is a raining event that occurs entirely within $\mathcal{I}$.

Once we have intervals in the semantics, we can add some tweaks to the framework and formulate clauses for phrases such as in 1984 . Assume that in addition to the interval in the index, there is a background interval $\mathcal{J}$. The initial value of $\mathcal{J}$ might be unrestricted - that is, $\mathcal{J}$ might be the full history of the world. Tweak the clauses for was and will so that they quantify over the intersection of $\mathcal{I}$ and $\mathcal{J}$. Then interpret in 1984 as restricting the background interval to 1984 .

A few extra tweaks are needed to model temporal indexicals such as yesterday - specifically, some explicit representation of the context of utterance. One option is to add an abstract representation of context to 
our point of evaluations. The standard, Kaplanian way to add context is to let $c$ be a parameter that records all the elements of context that are relevant to the interpretation of indexicals, and evaluate at points of the form $\langle\mathcal{M}, c, \mathcal{I}, t\rangle$. Then yesterday would set the background interval $\mathcal{J}$ to the day preceding the day of the context $c$ and set $t$ to some time within that day.

The addition of a context coordinate allows us to define some new concepts. As I mentioned, part of the job of semantics is to make predictions about the acceptability conditions of utterances in context. An important tool in that enterprise is a definition of truth at a context. The standard, Kaplanian approach to this stage of the theory is to stipulate that the parameters that go beyond the model and the context get their initial value assigned by the context of utterance. For example, one might say that $t_{\mathcal{M}, c}$ (more often written here as $t_{c}$ ) is the time at which context $c$ occurs (in model $\mathcal{M}$ ). In a setting with intervals, one would typically conceive of $\mathcal{I}_{\mathcal{M}, c}$ as a very narrow interval including only the moment of utterance. It is important to emphasize that this is an initial value: Tense operators might shift these parameters away from their initial values.

Having established these determination facts, we can characterize the relevant concept of truth at a context:

TRUTH AT A CONTEXT (WITH MODELS)

$\mathrm{A}$ is true in $c$ (relative to $\mathcal{M}$ ) iff $\llbracket \mathrm{A} \rrbracket^{\mathcal{M}, c}=\mathrm{I}$ iff $\llbracket \mathrm{A} \rrbracket^{\mathcal{M}, c, \mathcal{I}_{\mathcal{M}, c}}=\mathrm{I}$

As usual, omitting models improves legibility, so it's worth looking at that characterization again without them:

TRUTH AT A CONTEXT (WITHOUT MODELS)

$\mathrm{A}$ is true in $c$ iff $\llbracket \mathrm{A} \rrbracket^{c}=\mathrm{I}$ iff $\llbracket \mathrm{A} \rrbracket^{c, \mathcal{I}_{c}}=\mathrm{I}$

Adding context is an indispensable first move if our goal is to draw up a semantics for yesterday. The basic idea here might would be this: Past tense shifts the interval away from $\mathcal{I}_{c}$ to some prior interval $\mathcal{I}^{*}$ of evaluation, as demanded by the linear symmetric semantics with intervals. Then yesterday restricts that past interval to the day that precedes the day of the context of utterance. Fully implementing this idea and making sure it plays well with the many other desiderata that govern a theory of tense is beyond the scope of this discussion.

From a linguistic point of view, these variants of the linear symmetric semantics do not go nearly far enough. In her classic paper "Some Structural Analogies between Tenses and Pronouns" (1973), Barbara Partee (1973) 
argues that tenses behave in a way that seems importantly similar to pronouns. Partee notes examples like this:

(4) Sheila had a party last Friday and Sam sang a song

Example (4) illustrates that the time of the singing is anchored onto the time of the party. This anaphoric relationship is not explicable in a rigid system according to which tenses are existential quantifiers. By contrast, if tenses are allowed to perform pronoun-like reference to temporal points or intervals, we can make clearer sense of the temporal relationship between the two conjuncts of (4). This referential account is compatible with the view that tenses might sometimes also involve quantificational truthconditions. In particular, if was and will actually are object language quantifiers, there might be anaphoric relations between their domains. The referential account is also perfectly compatible with a symmetric approach to the meanings of predictive expressions, though it must be noted here that Partee herself has already blazed the trail for the modal revolution in the semantics of the future (Partee, 1973, pp. 60I-602).

\section{I.4 Temporal Ontology and Symmetric Semantics}

We have latched onto a core idea for a symmetric semantics of future and past, and developed a model theoretic treatment to go with it. I want to close this chapter on a different, more foundational note. How do these abstract model theories connect to the temporal reality that languages (even primitive ones like the one I set up) are meant to describe?

One way to approach this problem is by thinking about what our semantics tells us about truth-conditions. Our most general notion of truth is relativized to a model and a context. But the concept of truth that matters to "truth-conditional" semantics is not relativized to a model. It would be desirable to get rid of that relativization to models so that we could truly say that we have characterized the truth-conditions of some class of sentences. To bridge this gap, we must connect the set theoretic objects we call "models" with the temporal reality they represent.

In our specific application, we need to get clearer about how a temporally structured world might fix the parameters that define an abstract model. I will refer to this task as elaborating the fit of the representational models to reality. 
Linear models, such as the ones introduced in Definition I.I, naturally fit a metaphysics that treats past and future symmetrically. Metaphysical symmetry, as I understand it, is the requirement that future and past be alike in terms of ontology and structure. The ontology part of the requirement rules out, among other things, metaphysical views that deny future ontology (i.e., the existence of future objects and events) but do not deny past ontology. This is the growing block doctrine (Broad, I923; Tooley, 1997; Briggs and Forbes, 20I2). As for the structure part, it is the requirement that whatever structural constraints govern the temporal precedence relation must apply when the relation is reversed. For example, structural symmetry requires that if reality does not branch toward the past, it should not branch toward the future. This rules out the branching time metaphysics, which posits that reality branches toward the future but not toward the past (see Chapter 2). ${ }^{9}$

Although the requirements of ontological and structural symmetry are non-trivial, they do not pin down a unique conception of the nature of temporal reality. Fundamentally different conceptions of the nature of time are consistent with both requirements. A prototypical symmetric theory is the standard version of the block universe theory. According to this metaphysical conception, past, present, and future are all equally real; there is no objective present moment and no branching. ${ }^{\text {IO }}$ Another type of theory that is ontologically and structurally symmetrical is the moving spotlight theory, which adds to the block universe an objectively distinguished moment - an objective present. Even presentism - the view that only the present is real - could be developed so as to satisfy the two symmetry requirements.

Among these theories, the simplest fit with symmetric semantics is offered by the eternalist theories, which accept both future and past ontology (such as the block universe theory and the moving spotlight theory). Let us focus our presentation on these theories. What needs to be explained

9 This asymmetry is structural as opposed to ontological in the sense that a branching time metaphysician need not say that there is a difference in ontological status between future object and events and past objects and events.

Io The "no branching" condition is not definitional of the block universe theory - it is merely typical of its standard versions. There is nothing in principle that prevents us from thinking that the universe is a block and that it branches. A block universe theorist believes that the block is treelike, as long as there is no privileged point on the block. This point is made in many places, including by Cameron (2015), who is not himself a block theorist. 
is how, given a future-directed sentence in a given context, a theorist might use the abstract model theory to evaluate that sentence as true or false in that context. Suppose, for definiteness, that the temporal structure of $w$ is as an ordering of instantaneous moments without endpoints in either direction. Roughly speaking, moments are instantaneous snapshots of a world, three-dimensional Euclidean spaces characterizing a "frozen frame" of reality. ${ }^{\text {II }}$

For each point in time, moments settle the totality of categorical nontemporal facts that hold at that time. For example, a moment should settle whether Alaa is sick in her bed, whether she is asleep, whether she is a student, whether she is within one mile of her favorite ice-cream shop, and so on. It should not settle whether Carl has had a haircut on one of the previous three days, or whether he will walk to guitar lessons within the next hour, and so on.

Under this conception, an event of utterance can fix all the parameters that go in the abstract stipulation of a model. To illustrate this, consider our simplest linear models: triples of the form $\langle\mathcal{T},<, v\rangle$, consisting of a set of times, an ordering over it, and a valuation function. Consider an utterance event $e$ of some future-directed sentence $s ; e$ must take place in a unique, temporally structured world $w$. Suppose for simplicity that $w$ is a countably infinite succession of moments with no endpoints in either direction. Then we can choose $\mathcal{T}$ as the set of integers and $<$ as the less-than relation over $\mathcal{T}$. As a result, there is a one-to-one correspondence between $\mathcal{T}$ and the moments in $w$. Moreover, there is a one-to-one correspondence that respects the order of the moments in $w$. Call this order-preserving correspondence $h$.

Finally, set up the valuation function corresponding to $w$. Recall that we assumed that moments settle all the categorical tenseless facts at each time in world $w$. With that understanding, let $v$ map a time $t \in \mathcal{T}$ and a sentence radical $\mathrm{A}$ to $\mathrm{I}$ if $\mathrm{A}$ holds at $h(x)$ in $w$, where $h(t)$ is the image of time $t$ under the one-to-one correspondence between the points in $\mathcal{T}$ and the moments in $w$.

The moral of this exercise is that truth-conditional semanticists aiming to tell a complete story about the truth-conditions of temporal discourse must step outside the shell of the model theory and work carefully on how their model theory might connect with the underlying metaphysics.

II The terminology and the conception of "moments" comes from Belnap (I992), who views this correctly in my view - as an idealization that substantively simplifies the semantics. 


\section{Appendix: The Logic $K_{t}$}

To state the logic of standard tense operators correctly, we must rework some of our key definitions to better fit the standard approach in the semantics of modal logic.

Definition 1.3 (Temporal frames and models)

(i) A simple temporal frame is a pair $\langle\mathcal{T},<\rangle$ with $\mathcal{T}$ a set of times and $<$ a linear order on $\mathcal{T}$.

(ii) A simple temporal model $\mathcal{M}$ is a triple $\langle\mathcal{T},<, v\rangle$ with $\mathcal{T}$ a set of times; $<$ a linear order on $\mathcal{T}$; and $v$ a valuation function.

(iii) Given a frame $\mathcal{F}=\langle\mathcal{T},<\rangle$ and a model $\mathcal{M}=\left\langle\mathcal{T}^{\prime},<^{\prime}, v\right\rangle$, say that $\mathcal{M}$ is based on $\mathcal{F}$ iff $\mathcal{T}=\mathcal{T}^{\prime}$ and the orders $<$ and $<^{\prime}$ are also identical.

\section{Definition I.4 (Validity)}

(i) $\mathrm{A}$ is valid in model $\mathcal{M}$ iff for every $t \in \mathcal{T}_{\mathcal{M}}, \llbracket \mathrm{A} \rrbracket^{\mathcal{M}, t}=\mathrm{I}$.

(ii) $\mathrm{A}$ is valid in a frame $\mathcal{F}$ iff $\mathrm{A}$ is valid in every model $\mathcal{M}$ that is based on $\mathcal{F}$.

(iii) $\mathrm{A}$ is valid in the class of simple temporal frames iff $\mathrm{A}$ is valid in every simple temporal frame.

When $\mathrm{A}$ is valid in the class of simple temporal frames, write $=_{S T F} \mathrm{~A}$.

To axiomatize the class of valid sentences, it helps to consider the universal duals of will and was. So let

- $\triangleright \mathrm{A}={ }_{d f} \neg$ will $\neg \mathrm{A}$

- $\triangleleft \mathrm{A}={ }_{d f} \neg w a s \neg \mathrm{A}$

The intuitive interpretations of these operators are, respectively, always in the future and always in the past. The resulting logic $K_{t}$ is presented here axiomatically. Start with standard axioms for classical sentential logic, and add the following axiom schemas:

$$
\begin{aligned}
& \mathrm{K}_{\triangleright} . \triangleright(\mathrm{A} \supset \mathrm{B}) \supset(\triangleright \mathrm{A} \supset \triangleright \mathrm{B}) \\
& \mathrm{K}_{\triangleleft} . \triangleleft(\mathrm{A} \supset \mathrm{B}) \supset(\triangleleft \mathrm{A} \supset \triangleleft \mathrm{B}) \\
& \text { FB. } \mathrm{A} \supset(\triangleright \text { was } \mathrm{A}) \\
& \text { BF. } \mathrm{A} \supset(\triangleleft \text { will } \mathrm{A})
\end{aligned}
$$

Let ' $\mid-K_{t}$ ' denote the property of theoremhood in this system. The theorems of $K_{t}$ are the class of sentences defined recursively by starting with all the instances of all the axioms and by closing under the following rules:

MP. if $\left.\right|_{K_{t}} \mathrm{~A}$ and $\left.\right|_{K_{t}} \mathrm{~A} \supset \mathrm{B}$ then $\vdash_{K_{t}} \mathrm{~B}$ 
$\mathrm{NEC}_{\triangleright}$ if $\mid-K_{K_{t}} \mathrm{~A}$, then $\mid-K_{t} \triangleright \mathrm{A}$

$\mathrm{NEC}_{\triangleleft}$ if $\left.\right|_{K_{t}} \mathrm{~A}$, then $\left.\right|_{K_{t}} \triangleleft \mathrm{A}$

This logic is sound and complete with respect to the class of simple temporal frames.

Theorem I.5 The logic $K_{t}$ characterizes the class of simple temporal frames, i.e.,

$$
\vdash_{K_{t}} A \text { iff }=_{S T F} A
$$

